\title{
Chapter
}

\section{Cannabis and cognition: short- and long-term effects}

\author{
Nadia Solowij and Nicole Pesa
}

Twenty years ago cannabis was generally perceived to be a benign drug with few significant adverse effects. As outlined elsewhere in this book, evidence has since mounted in the scientific literature for a range of harms associated with the use of cannabis, including the development of dependence and health-related harms (see also Hall and Solowij, 1998; Hall and Degenhardt, 2009). As the overall theme of this book indicates, an association between cannabis use and the development of psychotic symptoms or overt psychosis has grown to be recognized as a significant potential harm, and investigating the mechanisms by which cannabis may trigger psychosis is a priority. This includes understanding the effects of cannabis on brain structure, biology and function. We recently highlighted a similarity between the cognitive impairment that has been reported in cannabis users and the deficits observed in schizophrenia (Solowij and Michie, 2007), suggesting common underlying neuropathology. Few would argue that cognition is not impaired to some degree during acute intoxication with cannabis. That impaired cognition persists beyond the period of acute intoxication is more contentious. Despite objective appraisals of the literature in interpreting the evidence, it is inevitable that researchers will be influenced by the weight of their own data in formulating scientific opinion. Accordingly, and on the basis of the accumulating evidence, this review will come to some rather different conclusions from those made in the first edition of this book (Pope and Yurgelun-Todd, 2004).

The goal of this chapter is to update our knowledge of the short- and long-term effects of cannabis on cognition based on integrating evidence from the most recent literature on this topic. We acknowledge the weight of evidence from our own studies that must inevitably guide us to the conclusions that we draw, while also aiming objectively to assess the evidence from multiple sources. We consider evidence from preclinical research, studies of acute administration of cannabinoids to humans, studies of long-term or heavy cannabis users tested in the unintoxicated state, including adults and adolescents and patients with schizophrenia, and we evaluate the evidence for recovery of function after prolonged abstinence.

\section{Animal studies}

A wealth of preclinical research shows an unequivocal role for the endogenous cannabinoid system in attention, memory, inhibitory control and multiple other cognitive processes, and that these are impaired following both acute and chronic cannabinoid administration (Egerton et al., 2006; Solowij and Michie, 2007; Pattij et al., 2008; Solowij and Battisti, 2008). Even a single administration of an ultra-low dose of $\Delta^{9}$-tetrahydrocannabinol (THC) (0.001-0.002 mg/ $\mathrm{kg}$ ) has been shown to result in long-term cognitive impairments in mice ( 3 weeks to 4 months post-injection) (Tselnicker et al., 2007; Amal et al., 2010).

Recent animal research supports the notion that the developing brain is more susceptible to the acute and chronic effects of exogenous cannabinoids, particularly the hippocampus. As outlined in Chapter 7, evidence is building from studies in which animals have been exposed to cannabinoids prenatally or during the pubertal/adolescent period, with greater immediate adverse effects on cognition and behavior observed in comparison to animals exposed during adulthood, as well as such effects persisting into adulthood with no further cannabinoid exposure (Kang-Park et al., 2007; Schneider, 2008; Realini et al., 2009; Rubino et al., 2009).

A recent study reported that the endocannabinoid system is significantly altered by exposure to THC during early, middle and late adolescence in rats (Ellgren et al., 2008). The normal proportional ratio of

Marijuana and Madness, Second Edition, ed. David Castle, Robin M. Murray and Deepak Cyril D’Souza. Published by Cambridge University Press. (C) Cambridge University Press 2012. 
anandamide and 2-arachidonoyl glycerol (2-AG) in the prefrontal cortex (PFC) and the nucleus accumbens was reversed by exposure to THC, and anandamide levels were increased in the nucleus accumbens. These dynamic changes in the mesocorticolimbic endocannibinoid (eCB) system (ECS) were induced by intermittent exposure to THC, which emulates the pattern of use among teenagers.

\section{Short-term effects in humans}

Numerous studies have examined the acute effects of cannabis on human cognition. That cannabis induces perceptual distortions and impairs memory and concentration during acute intoxication is generally well accepted. However, a recent systematic review of the literature to 2007 identified considerable inconsistency across findings. Zuurman et al. (2009) examined the effects of acute administration of cannabis or THC to healthy volunteers from 165 studies utilizing 318 measures with the goal of identifying specific biomarkers of cannabis intoxication and central nervous system effects, and considering dose of THC administered. While functional impairment was observed across an extensive range of measures, few met the criteria for biomarkers in terms of consistency of effect. This may have been somewhat obscured by variability across multiple factors in the studies reviewed, including the nature of the subjects (varying in degree of experience with cannabis and hence tolerance) and the wide range of test measures. The most reliable biomarkers were increased heart rate and subjective effects. Dose-related decrements were observed in some domains (e.g. auditory/verbal delayed recall and recognition), less clear effects in others (e.g. immediate recall) and reverse effects (decreased decrements with higher doses) in yet others (e.g. working memory, digit-symbol substitution, focused selective attention, visuomotor control). Inhibition, reasoning/association and reaction time, while impaired, showed no consistent dose-response effect. Biphasic effects of lower versus higher doses were also observed and the authors highlighted that the pattern of effects supported a relaxing, sedating and reduced attention effect of THC at lower doses, and greater stimulatory and aggressive effects at higher doses. They further commented on the potential for additional reliable biomarkers within the domains of memory and motor functions if the wide range of tests and measures were standardized.
Despite a degree of inconsistency and complexity associated with biphasic dose effects and tolerance, cannabis has been shown in many studies to acutely impair attention, learning, short-term memory, working memory, executive function, abstract ability and decision making (Hall and Solowij, 1998; Solowij, 1998; Iversen, 2003; Fletcher and Honey, 2006; Ranganathan and D'Souza, 2006; Solowij and Michie, 2007; Hall and Degenhardt, 2009; Zuurman et al., 2009; Sewell et al., 2010; Solowij and Pesa, 2010). A revival of interest in examining the acute effects of cannabinoids on cognition in humans has been evident in recent years, with greater application of prospective, double-blind, placebo-controlled, cross-over designs, and with particular interest in understanding the psychotomimetic effects of cannabis. Here we summarize key findings, focusing predominantly on these most recent studies.

There have been growing concerns regarding the increasing potency of cannabis preparations (see Chapter 4). Many studies of acute administration have demonstrated dose-response effects whereby the greater the dose of THC, the greater the impairment. One recent study examined a range of doses of THC relevant to designer-grade cannabis in common use in Europe and the UK (eg. sinsemilla, nederweed), administered to regular but not daily users in the form of joints mixed with tobacco. There were linear decrements with increasing dose in reaction time and errors in attention, and short-term memory tasks and impaired motor control (Hunault et al., 2009).

A range of attentional processes is impaired by cannabis acutely. Impaired performance on sustained attention (eg. on continuous performance tasks), selective, focused and divided attention tasks, as well as in preattentive sensory memory have been demonstrated after acute administration of cannabis or THC to humans (Ilan et al., 2004; O'Leary et al., 2007; Hunault et al., 2009; Ramaekers et al., 2009). Accuracy, increased error rates and slowed reaction times were shown in some studies to be dose-related. Ramaekers and colleagues (2009) found impaired performance on a divided attention task following high-dose $(500 \mu \mathrm{g} / \mathrm{kg})$ THC only in occasional, but not heavy users, suggesting tolerance. In contrast, both occasional and heavy users exhibited inhibitory control deficits in a "Stop Signal" task. Altered inhibitory processing is evident following acute intoxication, in particular through impulsive responding (Hart et al., 2001; McDonald et al., 2003). Imaging studies have found that THC-attenuated activation in 
the right inferior frontal and anterior cingulate cortex (ACC) (Borgwardt et al., 2008) and opposing effects of THC and cannabidiol (CBD) in the hippocampus were found during a "Go/NoGo" task (Bhattacharyya et al., 2009). A study of decision making, as assessed by the Iowa Gambling Task, found no disruption to risky behavior, only a slowing of performance in daily cannabis users during acute intoxication (Vadhan et al., 2007), while another found increased risky decision making and altered sensitivity to consequences after a higher dose of THC was given to occasional users (Lane et al., 2005b).

D'Souza and colleagues (2004) conducted a rigorous investigation of the effects of intravenous THC administered to healthy volunteers who had experience with cannabis use, but who were not heavy users. $\Delta^{9}$-Tetrahydrocannabinol induced transient positive and negative schizophrenia-like symptoms and impaired working memory, verbal memory, distractibility and verbal fluency. Similarly, Morrison et al. (2009) reported induction of positive psychotic symptoms and deficits in verbal episodic memory and executive function following administration of intravenous THC. Deficits in verbal learning and memory are perhaps the most robust impairments associated with acute cannabis use (Curran et al., 2002; D'Souza et al., 2004; Ilan et al., 2004; Morrison et al., 2009), with evidence of impaired immediate and delayed free recall of information, and difficulties in manipulating the contents of working memory, along with failure to use semantic processing and organization to optimize episodic memory encoding and impaired retrieval performance (Fletcher and Honey, 2006; Ranganathan and D'Souza, 2006).

Bhattacharyya and colleagues have reported a series of neuroimaging studies of the effects of orally administered THC or CBD (Bhattacharyya et al., 2009a; 2009b). They found that the effects of cannabis on verbal learning were mediated through its influence on left temporal activity (particularly parahippocampal), with modulation also of medial PFC and ACC activity during encoding or retrieval of information. $\Delta^{9}$-THC and CBD showed opposing effects in the striatum during verbal recall. These studies also elucidated the neural basis of the anxiogenic or anxiolytic effects of THC and CBD, respectively, as pertinent to understanding the propensity for cannabis to induce psychotic symptoms. Other recent neuroimaging studies of acute administration of cannabinoids have been reviewed by Martin-Santos et al. (2010) (see also Chapter 14).
Working memory is disrupted by acute cannabis use, with impaired performance, electroencephalogram (EEG) and event-related potential (ERP) measures (Ilan et al., 2004; D'Souza et al., 2004; Lane et al., 2005a). Regular but infrequent cannabis users showed dose-dependently impaired performance (greater errors) on a Sternberg memory task following acute administration of THC (O'Leary et al., 2007), and these have been associated with reduced frontal-midline EEG theta power (Bocker et al., 2007). Acute effects of cannabinoids on electrophysiology have also been demonstrated in infrequent cannabis users for the mismatch negativity (MMN) component of the ERP (MMN being an index of preattentive sensory memory) (Juckel et al., 2007) and the P300 component (an index of the allocation of attentional resources and updating of memory traces) (Roser et al., 2008).

Thus, further evidence has accumulated for a disruption of attention, memory and inhibitory control following acute administration of cannabis to humans, with some elucidation of the neural substrates of these effects, including evidence of differential effects of different cannabinoids (such as THC and CBD). It appears also that the response to acute cannabinoid administration is mediated by cannabis-use history and the development of tolerance to the acute effects in some cognitive tasks. However, more research is required to determine systematically the parameters of cannabis use that lead to the development of tolerance, the doses that may or may not elicit impaired performance in regular users and the cognitive tasks that are amenable to tolerance. For example, Boucher and colleagues (2009) showed that impairments in spatial working memory in rats are resistant to tolerance after extended administration of THC. We also do not know whether, or how, regular users may develop compensatory strategies during acute intoxication to facilitate performance that might otherwise be impaired. For example, in a risky decision-making task, Rogers et al. (2007) showed a reduction of risky behavior following lowdose sublingual administration of THC to healthy young adults (not regular cannabis users), with an adoption of more cautious cognitive strategies to compensate for the perceived disruption of effective decision making by cannabis. Thus regular users, due to their greater experience with cannabis, might be more likely to develop alternate compensatory strategies. 


\section{Long-term effects}

Studies of long-term and heavy cannabis users have continued to investigate residual or persistent effects of cannabis on cognitive function. Most studies have assessed cannabis users within 12-48 hours of last use of cannabis and cognitive impairment during this phase informs the functioning of regular users in the course of their daily lives when not acutely intoxicated. An increasing number of studies are applying longer periods of abstention, from 1 week through to 1 month or more. Some years ago, we postulated that the consequences of cannabis use may differ across the lifespan, with greater psychosocial, educational, maturational and mental health issues for adolescents and young adults, and cognitive deficits manifesting only after years of heavy cannabis use (Solowij and Grenyer, 2002). However, much evidence has now emerged for cognitive deficits to exist in younger cannabis users and interest has focused on the impact of cannabis use on the adolescent brain (see Chapter 7). Accordingly, we have structured this section to consider studies of adult cannabis users separately to those of cannabisusing adolescents and young adults who commenced cannabis use during early adolescence. We also consider briefly the growing literature on cognitive functioning in patients with schizophrenia who also use cannabis.

\section{Adult Studies}

\section{Attention}

Sustained attention, most often measured by continuous performance tasks (CPTs), is inconsistently impaired in chronic cannabis users (Pope et al., 2001; Indlekofer et al., 2009). However, even in the absence of overt performance deficits, lower glucose metabolism in orbitofrontal, temporal, hippocampal and parahippocampal regions has been observed during CPT performance in regular cannabis users (Voytek et al., 2005). Tonic alertness was impaired in moderate users (Indlekofer et al., 2009). A study of preattentive prepulse inhibition (PPI) attributed poor performance by chronic cannabis users to deficits in sustained attention, which were associated with greater frequency cannabis use (Scholes et al., 2009).

Selective and divided attention deficits in chronic cannabis users have been shown to be related to frequency and duration of long-term use, with only partial recovery after a mean of two years of abstinence
(Solowij, 1998). But even relatively light use (once a week) was related to some attentional dysfunction in young adults (Skosnik et al., 2001). The evidence suggests differential deficits associated with frequency versus duration of cannabis use, reflecting shorter- versus longer-lasting effects.

\section{Inhibition}

Impaired inhibitory processing, assessed through behavioral tasks such as the "Stroop, Go/NoGo" and a variety of decision-making and gambling tasks, is also impaired in long-term cannabis users (Bolla et al., 2002; Solowij et al., 2002; Solowij and Michie, 2007; Hester et al., 2009). Such tasks require the selection of an appropriate response while simultaneously inhibiting the inappropriate response. It has been suggested that the eCB system may modulate dopaminergic PFC and accumbal activity and contribute to inappropriate incentive salience to irrelevant stimuli; this may underlie attentional and inhibitory processing and decisionmaking deficits (Melis et al., 2004; Solowij and Michie, 2007; Pattij et al., 2008). Imaging studies show altered dorsolateral prefrontal cortical (DLPFC) and ACC activation during the interference condition of the Stroop task, despite reasonable task performance, in current cannabis users (Gruber and Yurgelun-Todd, 2005) and 1-month abstinent users (Eldreth et al., 2004). Performance on the Stroop task is inconsistently impaired in chronic cannabis users, but poorer performance has been associated with duration and dose, possibly interacting with low IQ and with altered electrophysiology (Bolla et al., 2002; Solowij et al., 2002; Battisti et al., 2010a). In chronic adult users with adequate inhibitory control performance, commission errors increased and a diminished capacity for behavior monitoring and error-awareness was associated with hypoactivity in the ACC and right insula (Hester et al., 2009).

\section{Working memory and other executive functions}

Working memory is the temporary encoding and manipulation of information that is a core component of executive functions of cognition. The involvement of the endogenous cannabinoid system in working memory has been well documented (Solowij and Michie, 2007; Pattij et al., 2008). A range of executive function tasks have been found to be impaired in both acute and chronic cannabis use (e.g. verbal fluency, Wisconsin Card Sorting Task, Ravens Progressive Matrices, Tower of London) (see Solowij and Michie, 
2007) but few studies have addressed working memory directly in cannabis users and this is an area that is receiving increasing interest. We have shown that chronic cannabis users are impaired on several measures from the Cambridge Neuropsychological Test Automated Battery (CANTAB), including Rapid Visual Information Processing, Pattern Recognition Memory, Spatial Recognition Memory, Spatial Span, Spatial Working Memory and Visuospatial Paired Associate Learning (Solowij et al., 2008). Abstinent cannabis users showed no performance deficits, but did demonstrate altered parietal brain activation in a Sternberg working memory task (Jager et al., 2006). Further neuroimaging studies indicate that cannabis users recruit additional brain regions in a compensatory manner in order to achieve adequate performance on working memory tasks (Kanayama et al., 2004; Martin-Santos et al., 2010).

In a recent study of verbal fluency, visual memory and short- and long-interval prospective memory thought to rely on executive functions, McHale and colleagues (2008) found that young adult cannabis users with recent use (past week) showed impaired memory function and generated fewer words than those abstinent longer than a week; both groups generated fewer words than non-user controls. The authors showed that these deficits were specific to cannabis use despite the fact that cannabis is often mixed with tobacco, as the deficits were not apparent in a tobacco-user control group. They suggested some recovery of cognitive ability with abstinence, but this may have been confounded by frequency of use as the "abstinent" group comprised twice-weekly users, whereas the "recent-use" group smoked five to six times per week.

\section{Verbal memory and other memory processes}

Verbal memory is consistently impaired in chronic cannabis users, with impaired performance on word list learning tasks (e.g. Rey Auditory Verbal Learning Task [RAVLT], the California Verbal Learning Task [CVLT] and Buschke's Selective Reminding Task). These studies have been extensively reviewed elsewhere, together with some early neuroimaging studies of verbal memory in cannabis users (Solowij and Michie, 2007; Solowij and Battisti, 2008). Overall, the evidence suggests that long-term or heavy cannabis users show impaired encoding, storage, manipulation and retrieval mechanisms. Users learn fewer words across trials and recall fewer words, particularly after interference or delay. Several studies have shown that these deficits are variously attributed to duration of cannabis use (Solowij et al., 2002; Messinis et al., 2006), frequency of use (Pope et al., 2001) or cumulative dosage effects (Bolla et al., 2002).

Recent neuroimaging studies have sought to elucidate the acute effects of THC and other cannabinoids (e.g. CBD) on neural substrates subserving verbal memory, as discussed above (Bhattacharyya et al., 2009; Martin-Santos et al., 2010), or attempted to relate brain structural changes in cannabis users to verbal memory deficits. For example, Yücel et al. (2008) found significantly reduced hippocampal volumes in longterm heavy cannabis users, who were also significantly impaired on the RAVLT, but memory performance was unrelated to hippocampal volumes. Such complex verbal learning tasks likely involve functional connectivity across a wide range of brain regions, and impaired performance is likely to be associated more with the functional activation of those regions, rather than their structure. A recent electrophysiological study in chronic users found poor word recall and alteration of the ERP-subsequent memory effect during encoding, a component thought to originate in the hippocampal region; this alteration was associated with a longer duration and an earlier onset of cannabis use (Battisti et al., 2010b).

More specific hippocampal-dependent tasks, such as pictorial-associative memory tasks, have also been investigated in 1-week abstinent cannabis users (Jager et al., 2007; Luijten et al., 2007). Task performance did not differ between moderately using young adults and non-user controls, but recall accuracy decreased as a function of exposure to cannabis and decreased activation was observed in users in bilateral parahippocampal regions and in the right DLPFC during learning (Jager et al., 2007). A study of hippocampaldependent face-name learning in young adult frequent users found impaired learning, short- and long-term memory and hypoactivation of frontal and temporal regions, with concomitant hyperactivation of parahippocampal regions during learning, reflective of both functional deficits and compensatory processes (Nestor et al., 2008). Similarly, Becker et al. (2010) found greater activation of the left parahippocampal gyrus during encoding in a face-profession associative-learning task in high- compared with low-frequency users; however, there were no apparent effects associated with duration of use, or age of onset of use. This too was interpreted as functional compensation to maintain performance. 


\section{Other cognitive functions}

Indlekofer et al. (2009), in a population-based study of moderate cannabis users, found deficits in prose recall (logical memory test) in association with lifetime cannabis use, and significantly increased self-reported cognitive failures (of memory, attention, perception and motor function) with more extensive cannabis use. Time estimation has been found to be altered during both acute intoxication and in some studies of chronic users (Solowij and Michie, 2007; Pattij et al., 2008). Typically, time is underestimated - the subjective experience is of time passing more slowly. Time estimation is thought to involve the cerebellum and chronic cannabis users have been shown to be impaired in a classical delayed eye-blink conditioning task that reflects cerebellar functional integrity (cerebellardependent associative learning) (Skosnik et al., 2008). Recent data suggest cerebellar structural alterations in chronic cannabis users (Solowij et al., 2011b).

Since cannabis alters mood during acute intoxication, interest has grown in exploring emotion and affect processing in chronic users. Gruber and colleagues (2009) examined regional brain activation to masked affective stimuli in heavy cannabis users and found altered frontal and limbic activity, with decreased activation of ACC and amygdala regions compared with controls, as well as differential effects for masked happy versus angry faces. Three studies of acute cannabis administration also found modulation of amygdala activity during processing of fearful faces, with opposing effects of THC and CBD (Phan et al., 2008; Bhattacharyya et al., 2009b; Fusar-Poli et al., 2009). We reported significantly reduced amygdala volumes in long-term heavy cannabis users (Yücel et al., 2008) but it is not yet known whether this is associated with emotional or affect processing deficits. Other recent neuroimaging research has examined reward processing mechanisms in chronic users, showing increased cerebellar and ventrostriatal activation during reward anticipation; the latter was correlated with the duration of cannabis use and lifetime dose of exposure (Nestor et al., 2010).

\section{Adolescent and early-onset young-adult studies}

Adolescence is the prime period for initiation of cannabis use and a significant proportion of adolescents use cannabis regularly. Adolescence is also a period of significant neural development, with resculpting of the brain in terms of pruning, apoptosis and myelination (Schneider, 2008). This suggests that substance use during this crucial neurodevelopmental period may impact brain maturation and plasticity, and an increasing body of evidence from animal research indicates that the adolescent brain is more vulnerable to some of the adverse effects of cannabis (Schneider, 2008; see also Chapter 7).

Two recent reviews have examined the literature on cognitive functioning specifically in adolescent cannabis users (Schweinsburg et al., 2008a; Jacobus et al., 2009). The primary findings from these reviews indicated evidence for impaired attention, processing speed, learning and memory, functional and subtle structural brain alterations and sleep disturbances in adolescents who use cannabis heavily. Further, it was suggested that cognitive deficits may persist for longer in adolescent users (6 weeks to 3 months) than has been shown in adult users, and particularly so in the domains of learning, memory and working memory. Here we highlight some of the recent findings in adolescent users.

\section{Attention}

Jacobsen and colleagues (2004) found that adolescent cannabis users made significantly more errors on a CPT task than non-using controls. Increased errors trended toward an association with greater exposure to cannabis. Early onset of cannabis use (i.e. before age 15 or 16 years) was found to be a strong predictor of attentional deficits during adulthood (Ehrenreich et al., 1999; Novaes et al., 2008). P300 amplitude, thought to reflect the allocation of attentional resources, has been found to be reduced in early-onset users (Kempel et al., 2003).

\section{Inhibition}

Poor performance on the Stroop task has been associated with early-onset cannabis use (Novaes et al., 2008; Battisti et al., 2010a). In a “Go/NoGo task", adolescent cannabis users' performance was adequate following 1 month abstinence, but altered activation was observed in frontal and parietal brain regions, with users requiring increased neural effort during the inhibition condition to maintain performance levels (Tapert et al., 2007). We found that adolescent cannabis use, but not alcohol use, was associated with increased risky and impulsive decision making, with users adopting strategies with higher levels of uncertainty and not utilising information effectively; also performance 
was related to an earlier onset of regular cannabis use (Solowij et al., in press).

\section{Working memory and executive functions}

Executive functions have been shown to be impaired in early-onset cannabis users (Pope et al., 2003), and adolescent users show a range of attention, working memory and executive function deficits on the CANTAB (Harvey et al., 2007). Performance on an n-back auditory working-memory task was shown to be impaired, as memory load increased in abstinent adolescent cannabis users, with some evidence of altered regional brain activation emerging during nicotine withdrawal (Jacobsen et al., 2007). Abstinent adolescent male cannabis users showed overactivity in prefrontal regions but no performance deficits in a Sternberg working memory task, and no alterations in an associative memory task (Jager et al., 2010). The authors suggested that their results supported the vulnerability of the developing frontal lobes to early-onset cannabis use. Two neuroimaging studies reported functional brain activation abnormalities in 28-day abstinent adolescents in a spatial working memory task and provide further evidence in these young users of the application of alternate strategies, and recruitment of additional brain regions in a compensatory manner in order to achieve adequate performance (Padula et al., 2007; Schweinsburg et al., 2008b).

\section{Verbal memory and other memory processes}

Verbal memory was found to be impaired in adolescent cannabis users (Harvey et al., 2007) and minimum 23-day abstinent adolescents, and associated with lifetime episodes of use (Medina et al., 2007). We have recently reported impaired verbal learning and memory in adolescent cannabis users compared with matched adolescent alcohol users and non-user controls and this was shown to increase with duration, quantity, frequency and age of onset of cannabis use and was unrelated to alcohol use (Solowij et al., 2011a). Importantly, an effect of earlier age of onset of cannabis use was retained after controlling for the extent of exposure to cannabis. This young sample had only moderate exposure to cannabis over $2-3$ years, yet showed impairment relative to their age-matched counterparts similar to that seen in adults with greater than 20 years of heavy use; we previously reported no such impairment in heavy adult users with 10 years use (Solowij et al., 2002). These robust findings indicate that cannabis adversely affects the developing brain and reinforce concerns regarding the impact of early exposure and the greater vulnerability of the adolescent brain.

Prospective memory has also been demonstrated to be impaired in adolescent and young adult users (Bartholomew et al., 2008; McHale et al., 2008). Altered electrophysiology during encoding of words was associated with an earlier onset of use in an adult sample (Battisti et al., 2010b). In contrast to decreased parahippocampal and DLPFC activation during learning in adult cannabis users, a study of adolescents found increased activation in the fusiform/parahippocampal area, inferior frontal gyrus, DLPFC, superior parietal cortex and the ACC (Luijten et al., 2007) suggestive of increased neural effort.

\section{Recovery of function with abstinence}

While an increasing number of studies have now assessed adult and adolescent cannabis users following abstinence of several weeks, very few have been specifically designed to determine whether functioning recovers. The study frequently cited as being definitive was that by Pope and colleagues (2001) that showed impaired memory function at baseline and after 7 days abstinence, but an apparent full recovery after 28 days abstinence (Pope et al., 2001). Delayed recall was still impaired relative to controls in analyses that did not adjust for verbal IQ differences, and in a reanalysis of their data, these authors found that those participants with an earlier onset of cannabis use were less likely to show full recovery (Pope et al., 2002). The participants in Pope et al.'s (2001) study remained in the general community for the course of the study, with abstinence monitored by the provision of urine samples. In another study using a similar verbal learning and memory test, participants were not assessed at baseline, but were admitted to an inpatient unit for supervised abstinence of 28 days before neuropsychological assessment (Bolla et al., 2002). This study found that memory deficits persisted and were dose-related, and similar decrements were also observed on tests of executive function, psychomotor speed and manual dexterity. Our own data from cannabis users engaged in a 4-month treatment program not aimed at abstinence (Solowij et al., 2002) suggest partial recovery with cessation or reduction of use (unpublished data). Adolescent cannabis users were also shown to be impaired in memory, attention, psychomotor speed and planning ability after 23 or more days of urine monitored abstinence; poor performance 
was shown to be a function of lifetime episodes of cannabis use after controlling for lifetime alcohol use (Medina et al., 2007). These findings suggest that cognitive deficits may indeed persist for a significant period beyond last use of cannabis and it is not known how long it may take before deficits recover and whether this may differ between adult and adolescent users.

A subset of participants from Pope et al.'s (2001) study were also found to show diminished activation in motor cortical circuits (Pillay et al., 2008) and persistent alterations of cerebral blood flow in the temporal lobe and cerebellum after 28 days abstinence (Sneider et al., 2008). Altered flow in frontal regions was apparent after 7 days abstinence but not 28 days, suggesting gradual normalization of neural activity in some regions but not others. Other neuroimaging studies have also reported functional activation differences in cannabis users after 7 days (Jager et al., 2006; 2007), 25 days (Eldreth et al., 2004; Bolla et al., 2005) or $>2$ months abstinence (Chang et al., 2006). We found impaired electrophysiological measures of selective attention in users who had been abstinent for an average of 2 years (Solowij, 1998). Clearly, further research is required to clarify the extent and time course of recovery of function after cessation of cannabis use.

\section{Patients with schizophrenia}

As discussed elsewhere in this book (Chapters 19, 20) cannabis exerts greater adverse effects on cognition when administered to patients with schizophrenia than it does in healthy individuals (D'Souza et al., 2008). This section will briefly review the evidence from the growing body of studies that have examined long-term effects on cognition in patients with schizophrenia who also use cannabis.

Since long-term or heavy cannabis use generally impairs cognition in otherwise healthy users, it might be expected that people with schizophrenia who are already cognitively impaired may be even more vulnerable to the adverse effects of cannabis on cognition. Surprisingly, the evidence to date has suggested the reverse (Potvin et al., 2008). Løberg and Hugdahl (2009) reviewed 23 recent studies that included a range of samples with psychosis and substance use (primarily cannabis) and found that 14 of these reported better cognition in the cannabis-using patient groups than in their non-using counterparts. More recently, we conducted a meta-analysis of 10 studies of cognition comprising 572 patients with established schizophrenia with and without cannabis use and found that patients with a history of cannabis use had superior neuropsychological functioning (Yücel et al., 2010). However, we observed that these findings were driven more so by those studies that included patients with any history of cannabis use, than by studies of patients with current or recent use. We also reported data from a first-episode sample and found that, relative to healthy controls, patients who used cannabis showed only selective neuropsychological impairment, while those without cannabis use had generalized deficits. Previous conjecturing of better premorbid functioning in cannabisusing patients has not been borne out in most studies that have considered this, but current explanations for better functioning in cannabis-using patients suggest that these findings may be driven by a subgroup of neurocognitively less-impaired patients who only developed psychosis after a relatively early initiation into cannabis use. Thus cannabis may cause a transient cognitive breakdown associated with the development of psychosis among less cognitively vulnerable individuals who might, in the absence of cannabis use, never have developed schizophrenia (Løberg and Hugdahl, 2009; Schnell et al., 2009; Yücel et al., 2010).

Notall studies reportbetter cognition in clinicalsamples. For example, Ringen and colleagues (2009) found some evidence of better cognition in cannabis-using patients with bipolar disorder, but significantly worse cognition in cannabis-using patients with schizophrenia, with relatively low-level cannabis use. Our own data in a small sample of chronic schizophrenia patients with extensive cannabis use (22 years, near daily, 5 joints/day) found little difference in neuropsychological functioning compared with non-using counterparts, although performance on some measures appeared to worsen with the extent of exposure to cannabis (Grenyer et al., 2010). However, the patients with extensive cannabisuse histories showed significant alterations in cerebellar white matter (Solowij et al., 2010b) and in hippocampal shape (Solowij et al., 2010). Clearly, the impact of cannabis use on brain function and structure in schizophrenia warrants further investigation.

\section{Conclusions}

A range of cognitive functions, encompassing attentional, memory, executive and inhibitory processes, are impaired during both the acute intoxication period and following long-term use of cannabis. There has been some elucidation of the neural substrates underlying 
these cognitive impairments. Cannabis users, and in particular regular users, may employ compensatory strategies to aid performance or require increased neural effort to maintain performance on certain tasks that may otherwise have been impaired. Cannabis-use history and the development of tolerance may mediate these effects.

Cognitive dysfunction in long-term or heavy cannabis users has been shown to increase as a function of frequency, duration, dose and age of onset of cannabis use. Recent interest has been directed toward cannabis use during adolescence, and evidence from animal and human studies suggests that the adolescent brain is more susceptible to the adverse effects of cannabis. Adolescent cannabis users show similar deficits to those observed in adult users, but greater cognitive impairment is evident the earlier that cannabis use commences. Cognitive dysfunction in long-term users tends to persist for at least one month following the cessation of cannabis use, and may persist for longer in adolescents, but the literature in both populations regarding extent of persistence is not definitive, although it is likely that deficits recover following prolonged abstinence.

Similarities between cognitive deficits in cannabis users and in people with schizophrenia, together with an overlap in brain morphological changes observed in each population (see Chapter 10), suggest that further research into the cognitive effects of cannabis may inform the mechanisms by which cannabis triggers symptoms of psychosis. The endogenous cannabinoid system modulates cognition and is altered in schizophrenia. Individual differences and variability in response to cannabis, during both acute intoxication and in the long-term, dictates a need to understand the mechanisms that constitute increased risk or susceptibility to both the adverse effects of cannabis on cognition and the development of psychosis. Further attention should be given to genetic variation, neurodevelopmental processes, and to the differential opposing or interactive effects of cannabinoids. When humans consume cannabis, they expose themselves not only to THC but also CBD and multiple other compounds that may exacerbate or diminish the effects of THC on the brain (See Chapters 1,2).

\section{Acknowledgements}

This review was supported by funding from the National Health and Medical Research Council (NHMRC) (Project Grants 459111 and 514604). Portions of the original research described here were supported by grants from the Clive and Vera Ramaciotti Foundation, the Schizophrenia Research Institute using infrastructure funding from NSW Health, and the University of Wollongong.

\section{References}

Amal, H., Fridman-Rozevich, L., Senn, R., et al. (2010) Long-term consequences of a single treatment of mice with an ultra-low dose of $\Delta 9$-tetrahydrocannabinol (THC). Behav Brain Res, 206:245-53.

Bartholomew, J., Holroyd, S. and Heffernan, T.M. (2008) Does cannabis use affect prospective memory in teenagers and young adults? Eur Psych, 23:S305.

Battisti, R.A., Roodenrys, S., Johnstone, S., et al. (2010a) Chronic cannabis users show altered neurophysiological functioning on Stroop task conflict resolution. Psychopharmacology.

Battisti, R.A., Roodenrys, S., Johnstone, S.J., et al. (2010b) Chronic use of cannabis and poor neural efficiency in verbal memory ability. Psychopharmacology, 209:319-40.

Becker, B., Wagner, D., Gouzoulis-Mayfrank, E., et al. (2010) Altered parahippocampal functioning in cannabis users is related to the frequency of use. Psychopharmacology, 209:361-74.

Bhattacharyya, S., Fusar-Poli, P., Borgwardt, S., et al. (2009a) Modulation of mediotemporal and ventrostriatal function in humans by $\Delta^{9}$-tetrahydrocannabinol: A neural basis for the effects of cannabis sativa on learning and psychosis. Arch Gen Psy, 66:442-51.

Bhattacharyya, S., Morrison, P., Fusar-Poli, P., et al. (2009b) Opposite effects of $\Delta^{9}$-tetrahydrocannabinol and cannabidiol on human brain function and psychopathology. Neuropsychopharmacol, 35:764-74.

Bocker, K.B.E., Gerritsen, J., Kruidenier, M., et al. (2007) Smoking high potency marijuana: theta power correlates with memory search. Eur Neuropsychopharmacology, 17, S553.

Bolla, K.I., Brown, K., Eldreth, D., et al. (2002) Dose-related neurocognitive effects of marijuana use. Neurology, 59:1337-43.

Bolla, K.I., Eldreth, D.A., Matochik, J.A., et al. (2005) Neural substrates of faulty decision-making in abstinent marijuana users. Neuroimage, 26:480-92.

Borgwardt, S.J., Allen, P., Bhattacharyya, S., et al. (2008) Neural basis of Delta-9-tetrahydrocannabinol and cannabidiol: effects during response inhibition. Biol Psychiatry, 64:966-73.

Boucher, A.A., Vivier, L., Metna-Laurent, M., et al. (2009). Chronic treatment with Delta(9)tetrahydrocannabinol impairs spatial memory and reduces zif268 expression in the mouse forebrain. Behav Pharmacol, 20:45-55. 
Chang, L., Yakupov, R., Cloak, C., et al. (2006). Marijuana use is associated with a reorganized visual-attention network and cerebellar hypoactivation. Brain, 129:1096-112.

Curran, H.V., Brignell, C., Fletcher, S., et al. (2002). Cognitive and subjective dose-response effects of acute oral $\Delta$ 9-tetrahydrocannabinol (THC) in infrequent cannabis users. Psychopharmacology, 164:61-70.

D'Souza, D.C., Abi-Saab, W.M., Madonick, S., et al. (2005). Delta-9-tetrahydrocannabinol effects in schizophrenia: implications for cognition, psychosis, and addiction. Biol Psychiatry, 57:594-608.

D'Souza, D.C., Perry, E., MacDougall, L., et al. (2004) The psychotomimetic effects of intravenous delta9-tetrahydrocannabinol in healthy individuals: Implications for psychosis. Neuropsychopharmacology, 29:1558-72.

Egerton, A., Allison, C., Brett, R.R., et al. (2006) Cannabinoids and prefrontal cortical function: Insights from preclinical studies. Neurosci Biobehav Rev, 30:680-95.

Ehrenreich, H., Rinn, T., Kunert, H.J., et al. (1999) Specific attentional dysfunction in adults following early start of cannabis use. Psychopharmacology, 142:295-301.

Eldreth, D.A., Matochik, J.A., Cadet, J.L., et al. (2004) Abnormal brain activity in prefrontal regions in abstinent marijuana users. Neuroimage, 23:914-20.

Ellgren, M., Artmann, A., Tkalych, O., et al. (2008) Dynamic changes of the endogenous cannabinoid and opioid mesocorticolimbic systems during adolescence: THC effects. European Neuropsychopharmacol, 18:826-34.

Fletcher, P.C. and Honey, G.D. (2006) Schizophrenia, ketamine and cannabis: Evidence of overlapping memory deficits. Trends $\operatorname{Cog} S c i, 10: 167-74$.

Fusar-Poli, P., Crippa, J.A., Bhattacharyya, S., et al. (2009) Distinct effects of (delta)9-tetrahydrocannabinol and cannabidiol on neural activation during emotional processing. Arch Gen Psych, 66:95-105.

Grenyer, B., Respondek, C., Gordon, S., et al. (2010) Cognitive, executive and social functioning in schizophrenia with and without comorbid cannabis use. Schizophrenia Res, 117:425.

Gruber, S.A., Rogowska, J. and Yurgelun-Todd, D.A. (2009) Altered affective response in marijuana smokers: An fMRI study. Drug Alcohol Depend, 105:139-53.

Gruber, S. and Yurgelun-Todd, D. (2005) Neuroimaging of marijuana smokers during inhibitory processing: a pilot investigation. Brain Res Cogni Brain Res, 23:107-18.

Hall, W. and Degenhardt, L. (2009) Adverse health effects of non-medical cannabis use. Lancet, 374:1383-91.

Hall, W. and Solowij, N. (1998) Adverse effects of cannabis. Lancet, 352:1611-16.

Hart, C.L., van Gorp, W., Haney, M., et al. (2001) Effects of acute smoked marijuana on complex cognitive performance. Neuropsychopharmacology, 25:757-65.
Harvey, M.A., Sellman, J.D., Porter, R.J., et al. (2007) The relationship between non-acute adolescent cannabis use and cognition. Drug Alcohol Rev, 26:309-19.

Hester, R., Nestor, L. and Garavan, H. (2009) Impaired error awareness and anterior cingulate cortex hypoactivity in chronic cannabis users. Neuropsychopharmacology, 34:2450-8.

Hunault, C.C., Mensinga, T.T., Böcker, K.B.E., et al. (2009) Cognitive and psychomotor effects in males after smoking a combination of tobacco and cannabis containing up to $69 \mathrm{mg}$ delta-9-tetrahydrocannabinol (THC). Psychopharmacology, 204:85-94.

Ilan, A.B., Smith, M.E. and Gevins, A. (2004) Effects of marijuana on neuropsychological signals of working and episodic memory. Psychopharmacology, 176:214-22.

Indlekofer, F., Piechatzek, M., Daamen, M., et al. (2009) Reduced memory and attention performance in a population-based sample of young adults with a moderate lifetime use of cannabis, ecstasy and alcohol. J Psychopharmacology, 23:495-509.

Iversen, L. (2003) Cannabis and the brain. Brain, 126:1252-70.

Jacobsen, L.K., Mencl, W.E., Westerveld, M., et al . (2004) Impact of cannabis use on brain function in adolescents. Ann NY Acad Sci, 1021:384-90.

Jacobsen, L.K., Pugh, K.R., Constable, R.T., et al. (2007) Functional correlates of verbal memory deficits emerging during nicotine withdrawal in abstinent adolescent cannabis users. Biol Psychiatry, 61:31-40.

Jacobus, J., Bava, S., Cohen-Zion, M., et al. (2009) Functional consequences of marijuana use in adolescents. Pharmacol Biochem Behav, 92:559-65.

Jager, G., Block, R.I., Luijten, M., et al. (2010) Cannabis use and memory brain function in adolescent boys: A cross-sectional multicenter functional magnetic resonance imaging study. J Am Acad Child Adolesc Psychiatry, 49:561-72.

Jager, G., Kahn, R.S., Van den Brink, W., et al. (2006) Long-term effects of frequent cannabis use on working memory and attention: an fMRI study. Psychopharmacology (Berl), 185:358-68.

Jager, G., Van Hell, H.H., De Win, M.M.L., et al. (2007) Effects of frequent cannabis use on hippocampal activity during an associative memory task. Eur Neuropsychopharmacol, 17:289-97.

Juckel, G., Roser, P., Nadulskic, T., et al. (2007) Acute effects of $\Delta 9$-tetrahydrocannabinol and standardized cannabis extract on the auditory evoked mismatch negativity. Schizophr Res, 97:109-17.

Kanayama, G., Rogowska, J., Pope, H.G., et al. (2004) Spatial working memory in heavy cannabis users: a functional magnetic resonance imaging study. Psychopharmacology, 176:239-47. 
Kang-Park, M., Wilson, W.A., Kuhn, C.M., et al. (2007) Differential sensitivity of GABAA receptor-mediated IPSCs to cannabinoids in hippocampal slices from adolescent and adult rats. J Neurophysiol, 98:1223-30.

Kempel, P., Lampe, K., Parnefjord, R., et al. (2003) Auditoryevoked potentials and selective attention: different ways of information processing in cannabis users and controls. Neuropsychobiology, 48:95-101.

Lane, S.D., Cherek, D.R., Lieving, L.M., et al. (2005a) Marijuana effects on human forgetting functions. J Exp Anal Behav, 83:67-83.

Lane, S.D., Cherek, D.R., Tcheremissine, O.V., et al. (2005b) Acute marijuana effects on human risk taking. Neuropsychopharmacology, 30:800-9.

Løberg, E.M. and Hugdahl, K. (2009) Cannabis use and cognition in schizophrenia. Front Hum Neurosci, 3:53.

Luijten, M., Jager, G., Block, R.I., et al. (2007) Effects of cannabis use on associative memory in adolescents, and the role of personality measures: an fMRI study. Eur Neuropsychopharmacol, 17:S552-S553.

Martin-Santos, R., Fagundo, A.B., Crippa, J.A., et al. (2010) Neuroimaging in cannabis use: a systematic review of the literature. Psychol Med, 40:383-98.

McDonald, J., Schleifer, L., Richards, J.B., et al. (2003) Effects of THC on behavioral measures of impulsivity in humans. Neuropsychopharmacology, 28:1356-65.

McHale, S. and Hunt, N. (2008) Executive function deficits in short-term abstinent cannabis users. Human Psychopharmacol, 23:409-15.

Medina, K.L., Hanson, K.L., Schweinsburg, A.D., et al. (2007) Neuropsychological functioning in adolescent marijuana users: Subtle deficits detectable after a month of abstinence. J Int Neuropsychol Soc, 13:807-20.

Melis, M., Perra, S., Muntoni, A.L., et al. (2004) Prefrontal cortex stimulation induces 2-arachidonoyl-glycerolmediated suppression of excitation in dopamine neurons. J Neurosci, 24:10707-15.

Messinis, L., Kypriandou, A., Malefaki, S., et al. (2006) Neuropsychological deficits in long-term frequent cannabis users. Neurology, 66:737-9.

Morrison, P.D., Zois, V., McKeown, D.A., et al. (2009) The acute effects of synthetic intravenous Delta9tetrahydrocannabinol on psychosis, mood and cognitive functioning. Psychol Med, 39:1607-16.

Nestor, L., Hester, R. and Garavan, H. (2010) Increased ventral striatal BOLD activity during non-drug reward anticipation in cannabis users. Neuroimage, 49:1133-43.

Nestor, L., Roberts, G., Garavan, H., et al. (2008) Deficits in learning and memory: parahippocampal hyperactivity and frontocortical hypoactivity in cannabis users. Neuroimage, 40:1328-39.

Novaes, M.A.F.P., Guindalini, C., Almeida, P., et al. (2008) Cannabis use before age 15 years is associated with poorer attention and executive function. Biol Psychiatry, 63:18S-19S.

O’Leary, D.S., Block, R.I., Koeppel, J.A., et al. (2007) Effects of smoking marijuana on focal attention and brain blood flow. Human Psychopharmacol, 22:135-48.

Padula, C.B., Schweinsburg, A.D. and Tapert, S.F. (2007) Spatial working memory performance and fMRI activation interaction in abstinent adolescent marijuana users. Psychol Addict Behav, 21:478 - 87.

Pattij, T., Wiskerke, J. and Schoffelmeer, A.N. (2008) Cannabinoid modulation of executive functions. Eur J Pharmacol, 585:458-63.

Phan, K.L., Angstadt, M., Golden, J., et al. (2008) Cannabinoid modulation of amygdala reactivity to social signals of threat in humans. J Neurosci, 28:2313-9.

Pillay, S.S., Rogowska, J., Kanayama, G., et al. (2008) Cannabis and motor function: $\mathrm{fMRI}$ changes following 28 days of discontinuation. Exp Clin Psychopharmacol, 16:22-32.

Pope, H.G., Gruber, A.J., Hudson, J.I., et al. (2003) Earlyonset cannabis use and cognitive deficits: What is the nature of the association? Drug Alcohol Depend, 69:303-10.

Pope, H.G., Gruber, A.J., Hudson, J.I., et al. (2001) Neuropsychological performance in long-term cannabis users. Arch Gen Psychiatry, 58:909-15.

Pope, H.G., Gruber, A.J., Hudson, J.I., et al. (2002) Cognitive measures in long-term cannabis users. J Clin Pharmacol, 42:41S-47S.

Pope, H.G. and Yurgelun-Todd, D. (2004) Residual cognitive effects of long-term cannabis use. In Castle, D.J. and Murray, R.M. (Eds.), Marijuana and Madness, 1st ed. (pp. 198-210). Cambridge: Cambridge University Press.

Potvin, S., Joyal, C.C., Pelletier, J., et al. (2008) Contradictory cognitive capacities among substance-abusing patients with schizophrenia: a meta-analysis. Schizophr Res, 100:242-51.

Ramaekers, J.G., Kauert, G., Theunissen, E.L., et al. (2009). Neurocognitive performance during acute THC intoxication in heavy and occasional cannabis users. J Psychopharmacol, 23:266-77.

Ranganathan, M. and D'Souza, D.C. (2006) The acute effects of cannabinoids on memory in humans: a review. Psychopharmacology, 188:425-44.

Realini, N., Rubino, T. and Parolaro, D. (2009) Neurobiological alterations at adult age triggered by adolescent exposure to cannabinoids. Pharmacol Res, 60:132-8.

Ringen, P.A., Vaskinn, A., Sundet, K., et al. (2009) Opposite relationships between cannabis use and neurocognitive functioning in bipolar disorder and schizophrenia. Psychological Med, 6:1-11. 
Rogers, R.D., Wakeley, J., Robson, P.J., et al. (2007) Reinforcement processing in the risky decision-making of young healthy adults. Neuropsychopharmacology, 32:417-28.

Roser, P., Juckel, G., Rentzsch, J., et al. (2008) Effects of acute oral $\Delta^{9}$-tetrahydrocannabinol and standardized cannabis extract on the auditory P300 event-related potential in healthy volunteers. Eur Neuropsychopharmacology, 18:569-77.

Rubino, T., Realini, N., Braida, D., et al. (2009) Changes in hippocampal morphology and neuroplasticity induced by adolescent THC treatment are associated with cognitive impairment in adulthood. Hippocampus, 19:763-72.

Schneider, M. (2008) Puberty as a highly vulnerable developmental period for the consequences of cannabis exposure. Addict Biol, 13:253-63.

Schnell, T., Koethe, D., Daumann, J., et al. (2009) The role of cannabis in cognitive functioning of patients with schizophrenia. Psychopharmacology (Berl), 205: 45-52.

Scholes, K.E. and Martin-Iverson, M.T. (2009) Alterations to pre-pulse inhibition (PPI) in chronic cannabis users are secondary to sustained attention deficits. Psychopharmacology, 207:469-84.

Schweinsburg, A.D., Brown, S.A. and Tapert, S.F. (2008a) The influence of marijuana use on neurocognitive functioning in adolescents. Curr Drug Abuse Rev, 1:99-111.

Schweinsburg, A.D., Nagel, B.J., Schweinsburg, B.C., et al. (2008b) Abstinent adolescent marijuana users show altered fMRI response during spatial working memory. Psychiatry Res, 163:40-51.

Sewell, R.A., Skosnik, P.D., Garcia-Sosa, I., et al. (2010) Behavioral, cognitive and psychophysiological effects of cannabinoids: relevance to psychosis and schizophrenia. Rev Bras Psiquiatr, 32:S15-S30.

Skosnik, P.D., Edwards, C.R., O’Donnell, B.F., et al. (2008) Cannabis use disrupts eyeblink conditioning: Evidence for cannabinoid modulation of cerebellar-dependent learning. Neuropsychopharmacology, 33:1432-40.

Skosnik, P.D., Spatz-Glenn, L. and Park, S. (2001) Cannabis use is associated with schizotypy and attentional disinhibition. Schizophr Res, 48:83-92.

Sneider, J.T., Pope Jr, H.G., Silveri, M.M., et al. (2008) Differences in regional blood volume during a 28-day period of abstinence in chronic cannabis smokers. Eur Neuropsychopharmacol, 18, 612-19.

Solowij, N. (1998). Cannabis and cognitive functioning. Cambridge: Cambridge University Press.

Solowij, N. and Battisti, R. (2008) The chronic effects of cannabis on memory in humans: a review. Curr Drug Abuse Rev, 1:81-98.

Solowij, N. and Grenyer, B. (2002) Are the adverse consequences of cannabis use age-dependent? Addiction, 97:1083-6.
Solowij, N., Jones, K.A., Rozman, M.E., et al. (2011) Verbal learning and memory in adolescent cannabis users, alcohol users and non-users. Psychopharmacology, 216:131-144.

Solowij, N., Jones, K.A., Rozman, M.E., et al. (2011) Reflection impulsivity in adolescent cannabis users. Psychopharmacology, 24(3):A63.

Solowij, N. and Michie, P. (2007). Cannabis and cognitive dysfunction: Parallels with endophenotypes of Schizophrenia? J Psychiatry Neurosci, 32:30-52.

Solowij, N., and Pesa, N. (2010) Cognitive abnormalities and cannabis use. Rev Bras Psiquiatr, 32:S31-S40.

Solowij, N., Respondek, C., Battisti, R., et al. (2008). Visuospatial memory deficits in long term heavy cannabis users: relation to psychotic symptoms and regional brain volumes. Int J Neuropsychopharmacol, 11: 242 .

Solowij, N., Stephens, R.S., Roffman, R.A., et al. (2002). Cognitive functioning of long-term heavy cannabis users seeking treatment. J Am Med Assoc, 287:1123-31.

Solowij, N., Walterfang, M., Respondek, C., et al. (2010). Alterations of hippocampal shape in cannabis users with and without schizophrenia. Schizophr Res, 117:426.

Solowij, N., Yücel, M., Respondek, C., et al. (2011b) Cerebellar white matter changes in cannabis users with and without schizophrenia. Psychol Med. DOI: 10.1017/S003329171100050X.

Tapert, S.F., Schweinsburg, A.D., Drummond, S.P.A., et al. (2007) Functional MRI of inhibitory processing in abstinent adolescent marijuana users. Psychopharmacology, 194:173-83.

Tselnicker, I., Keren, O., Hefetz, A., et al. (2007) A single low dose of tetrahydrocannabinol induces long-term cognitive deficits. Neuroscience Letters, 411:108-11.

Vadhan, N.P., Hart, C.L., van Gorp, W.G., et al. (2007) Acute effects of smoked marijuana on decision making, as assessed by a modified gambling task, in experienced marijuana users. J Clin Exp Neuropsychol, 29:357-64.

Voytek, B., Berman, S.M., Hassid, B.D., et al. (2005) Differences in regional brain metabolism associated with marijuana abuse in methamphetamine abusers. Synapse, 57:113-15.

Yücel, M., Bora, E., Lubman, D.I., et al. (2010) The impact of cannabis use on cognitive functioning in patients with schizophrenia: A meta-analysis of existing findings and new data in a first-episode sample. Schizophrenia Bulletin, DOI:10.1093/schbul/sbq079.

Yücel, M., Solowij, N., Respondek, C., et al. (2008) Regional brain abnormalities associated with long-term heavy cannabis use. Arch Gen Psychiatry, 65:694-701.

Zuurman, L., Ippel, A.E., Moin, E., et al. (2009) Biomarkers for the effects of cannabis and THC in healthy volunteers. Brit J Clin Pharmacol, 67:5-21. 